\title{
ESCRIBIENDO LA GUERRA FRÍA LATINOAMERICANA: ENTRE EL SUR "LOCAL" Y EL NORTE "GLOBAL"
}

Escrevendo a Guerra Fria latino-americana: entre o Sul "local" e o Norte "global"

Writing the Latin American Cold War: between the "local" South and the "global" North

ALDO MARCHESI

http://dx.doi.org/10.1590/S2178-14942017000100010

Aldo Marchesi é mestre e doutor em História pela Universidade de Nova lorque e professor da Universidade da República (Uruguai) (aldomarchesi70@gmail.com).

Artigo recebido em 15 de dezembro de 2016 e aprovado para publicação em 2 de fevereiro de 2017.

Revisión de español de Óscar Curros. 


\section{RESUMEN}

Mi trabajo propone una agenda para posibles diálogos entre la historia reciente del cono sur y los estudios de la guerra fría. El campo de la historia reciente, escrito principalmente por historiadores del cono sur, relacionado con la historia de la segunda mitad del siglo XX, se ha centrado principalmente en los escenarios nacionales de polarización política y social que surgieron durante los años sesenta, Los regímenes y, por último, las luchas por el derecho humano para la democratización durante los años ochenta. Estos estudios históricos han innovado en sus métodos historiográficos, en las formas de concebir la relación entre cultura y política, y también en la comprensión de las tensiones entre el discurso histórico y las memorias públicas. Más allá de estas innovaciones, estos estudios parecen estar atrapados en las esferas nacionales sin intercambiar sobre las similitudes y circulación de ideas y personas que ocurrieron en la región. Por otro lado, los estudios sobre la guerra fría, escritos principalmente en Estados Unidos, que trabajaron en la región, se han centrado principalmente en el impacto de la política exterior estadounidense. Aunque estos estudios abordaron las dimensiones locales, se centraron principalmente en las cuestiones relacionadas con el papel de los Estados Unidos sin considerar la agencia de actores locales en este proceso global. Entre estos dos campos de estudio hay vacíos históricos relacionados con los espacios transnacionales de circulación horizontal de ideas y actores dentro de la región que han sido principalmente ignorados por ambas historiografías. A través de algunos ejemplos históricos sugeriré formas de cruzar ambos campos de estudios para enriquecer la reflexión sobre la guerra fría latinoamericana. Palabras Clave: Guerra Fría Latinoamericana; Historia Transnacional; Historia Global; Historiografía; Historia reciente.

\section{RESUMO}

Meu artigo propõe uma agenda para possíveis diálogos entre a história recente do cone sul e os estudos da guerra fria. 0 campo histórico recente, escrito principalmente por historiadores do cone sul, relacionado à história da segunda metade do século XX, tem focado principalmente os cenários nacionais de polarização política e social que surgiram nos anos 60 , a reação autoritária que instalou o terrorismo de Estado Regimes e, por fim, as lutas pelos direitos humanos para a democratização durante os anos oitenta. Esses estudos históricos têm inovado nos seus métodos historiográficos, nas formas de conceber a relação entre cultura e política, e também na compreensão das tensões entre o discurso histórico e as memórias públicas. Além destas inovações, esses estudos parecem estar presos nas esferas nacionais sem trocar as semelhanças e circulação de ideias e pessoas que aconteceram na região. Por outro lado, os estudos de guerra fria, escritos principalmente nos EUA, que trabalharam na região, concentraram-se principalmente no impacto das políticas externas dos EUA. Embora esses estudos tenham abordado as dimensões locais, eles se concentraram principalmente nas questões relacionadas ao papel dos EUA sem considerar a agência de atores locais nesse processo global. Entre esses dois campos de estudo, há vácuos históricos relacionados a espaços transnacionais de circulação horizontal de ideais e atores dentro da região que tem sido principalmente ignorado por ambas as historiografias. Através de alguns exemplos históricos vou sugerir maneiras de cruzar ambos os campos de estudos, a fim de enriquecer a reflexão sobre a guerra fria latino-americana.

PalaVRAS-CHAVE: Guerra Fria latino-americana; História Transnacional; História Global; Historiografia; História recente.

\section{Abstract}

My paper proposes an agenda for possible dialogues between the southern cone's recent history and the cold war studies. The recent history field, written mainly by southern cone's historians, related to the history of the second half of twenty century has focused mainly on the national scenarios of political and social polarization that emerged during the sixties, the authoritarian reaction that installed state terror regimes, and, lastly, the human right struggles for democratization during the eighties. These historical studies have innovated in their historiographical methods, in the ways to conceive the relationship between culture and politics, and also in the understanding of the tensions between historical discourse and public memories. Beyond these innovations these studies seems to be trapped in the national spheres without exchanging on the similarities and circulation of ideas and persons that happened within the region. On the other hand the cold war studies, written mainly in the US, that worked on the region has mainly focused on the impact of the US foreign policies. Although these studies addressed the local dimensions, they mainly focused on the questions related to the role of the US without considering the agency of local actors in this global process. Between these two fields of study there are historical vacuums related to transnational spaces of horizontal circulation of ideas and actors within the region that has been mainly disregarded by both historiographies. Through some historical examples I will suggest ways to intersect both fields of studies in order to enrich the reflection on the Latin-American cold war.

KeYwORDS: Latin American Cold War; Transnational History; Global History; Historiography; Recent History. 


\section{INTRODUCCIÓN: ¿PARA QUÊ LA HISTORIA GLOBAL?}

\section{$\mathrm{L}$} a llamada historia global ha tenido un desembarco relativamente tardío en la historiografía latinoamericana que se produce en la región e incluso en la que se produce en Estados Unidos. Los motivos son diversos. Por un lado, las historiografías nacionales se han enfocado en estudiar sus propios países. Aunque en la segunda mitad del siglo XX diferentes aproximaciones cuestionaron el peso de lo nacional y sugirieron perspectivas que interpelaban esta narrativa, ofreciendo miradas alternativas que tuvieron que ver con la clase, la raza, el género, la vida cotidiana, entre otros asuntos, estos enfoques se desarrollaron dentro de la unidad de análisis del Estado Nación. Salvo algunas excepciones, la mayoría de los historiadores, así como las instituciones que promovieron la investigación histórica, se concentraron en dicha unidad. Además de las inquietudes académicas, los recursos de nuestras universidades tampoco colaboraron a otro tipo de desarrollo. Como varios han dicho, hacer historia global es caro. Requiere realizar estancias de mediana duración en archivos que están en diferentes países y tener cierta habilidad y conocimiento de diferentes lenguas. Además, y tal vez mucho más importante, se necesitan bibliotecas que efectivamente tengan una pretensión global, 0 al menos regional.

Pero tal vez habría que invertir la pregunta. ¿Por qué tendría que llegar la historia global? Hace ya décadas que se vienen cuestionando las relaciones complejas en la que se estructura la geopolítica del conocimiento. Muchas veces, desde las periferias se asumen conductas imitativas, que simplemente terminan legitimando el saber producido en ciertos centros y reinstalando el lugar periférico de la periferia. En un mundo académico paradójico, que da una imagen positivamente globalizante debido a las posibilidades de circulación de recursos y personas, pero que a la vez es profundamente desigual en las condiciones de producción y reconocimiento, resulta necesario pensar reflexivamente acerca de la utilidad de la incorporación de ciertos enfoques para aquellos que trabajamos en esta parte del mundo, así como lo que nosotros tenemos para aportar a esa reflexión. ${ }^{1}$

En un trabajo reciente, Dominic Sanchsemmaier repasaba los desarrollos de tres países donde la historia global ha tenido desarrollos importantes: Estados Unidos, Alemania y China (Sanchsemmaier, 2011). En cada uno de los casos, la autora señala determinadas condiciones 
contextuales que impulsaron este tipo de enfoques. En dos casos se trata de las principales potencias del siglo XX, que libraron dos guerras por la hegemonía mundial. En el último, de un país continente, que se anticipa como la próxima potencia imperial en el siglo XXI. No estoy diciendo que para hacer historia global sea necesario tener una tradición imperial, pero lo cierto es que son países que, por diversos motivos históricos y presentes, han tenido que pensar y piensan más allá de sus fronteras nacionales. Nada de esto se puede encontrar en Latinoamérica, donde incluso no estamos hablando de un país, sino de un continente cuya propia identidad político-cultural ha estado en disputa entre el latinoamericanismo, el panamericanismo y otras aproximaciones más contemporáneas durante todo el siglo XX (Mignolo, 2007). Frente a esto, la pregunta de cuáles son las condiciones contextuales, así como la de la utilidad de incorporar la historia transnacional y global a nuestras perspectivas latinoamericanas, es relevante. Me anticipo a responder que mi respuesta será positiva. Pero me parece que, si tiene algún valor dicha respuesta, la misma está vinculada a los motivos y a la manera en que dicha noción debería ser incorporada, mas que en la mera respuesta afirmativa.

En What is Global History?, uno de los más recientes esfuerzos por sistematizar este enfoque, Sebastian Conrad señalaba algunas características que resumen las inquietudes metodológicas vinculadas a la historia global. En su perspectiva, los historiadores globales no están solamente preocupados con visiones macro, sino que buscan situar asuntos históricos concretos con un potencial global, lo que los lleva a experimentar con nociones alternativas del espacio. La tarea no se restringe a trabajar sobre una unidad histórica específica, sino a interrelacionar las diferentes escalas, que van desde lo más general a lo más particular, y solo pueden ser entendidas a través de dicha interacción. Esta consideración por la manera en que individuos y sociedades interactúan los lleva a abandonar explicaciones endógenas sobre los cambios. Además, los historiadores globales tienden a desestimar los enfoques teleológicos, que proponen una idea predeterminada acerca de la transición de la tradición a la modernidad. El cambio, se piensa en términos de sincronicidad entre diferentes aéreas del planeta. Todas estas preocupaciones también los llevan a una autoconciencia de las condiciones eurocéntricas del conocimiento, así como a una autorreflexión acerca de la posicionalidad de producción del pensamiento. Conrad reconoce que existen diferentes lugares donde se han desarrollado estas perspectivas. Y que el lugar desde el cual se investiga y se escribe dicha historia global es relevante para el tipo de preguntas y aproximaciones metodológicas que se realizan para pensarla (Conrad, 2015: 216). En dicho texto, Conrad sintetiza (2015: 89) la promesa última de la historia global como una perspectiva que logra trascender la dicotomía interno y externo para pensar los procesos históricos. En este sentido, comparte la idea que la historia global no está atada a una metodología restringida o a una aproximación teórica particular sobre lo posnacional, sino a una vocación empírica por 
seguir nuestros objetos de estudio mas allá de la nación, y que las maneras en que esa búsqueda se puede desarrollar son múltiples.

Aunque la producción académica latinoamericana de los años cincuenta y sesenta, vinculada a la tradición desarrollista y dependentista, es considerada como un posible antecedente de algunos enfoques contemporáneos de la historia global, el espacio de América Latina es escaso en la producción actual. Desde este lugar se ha escrito poco sobre historia global, y el continente ha tenido un lugar menor en la historia global. A modo de ejemplo, si uno repasa el Journal of Global History, vemos que son pocos los artículos que tratan sobre el continente. De todos modos, hay áreas del conocimiento donde la producción latinoamericana realizada bajo esta perspectiva de la historia global ha tenido desarrollos relevantes. Entre otros ejemplos, podemos nombrar los trabajos sobre esclavitud y la diáspora africana, las investigaciones sobre el mundo colonial, el proyecto de iberconceptos y, en las últimas décadas, los trabajos vinculados a la influencia cultural, política y social de los Estados Unidos en la región durante el siglo XX.

En relación con los estudios de la Guerra Fría desarrollados desde una perspectiva global, podríamos decir que se repite la lógica mencionada anteriormente acerca del lugar de América Latina. Con la excepción del trabajo ya clásico de Odd Arn Westad, The global Cold War: third world interventions and the making of our times, las principales referencias anteriores del período no tuvieron una mayor consideración de América Latina. ${ }^{2}$

\section{HISTORIA RECIENTE Y LOS ESTUDIOS SOBRE LA GUERRA FRÍA EN AMÊRICA LATINA}

$\mathrm{E}$ n las últimas dos décadas una generación de investigadores ha contribuido al desarrollo de la llamada historia reciente en el Cono Sur. En los diferentes países, estos investigadores han tomado el pasado de la segunda mitad del siglo XX asociado al ciclo de polarización social y política, el desarrollo de regímenes autoritarios y, posteriormente, las transiciones a la democracia y han innovado en las maneras de estudiar estos temas, que tradicionalmente habían sido estudiados por otros ámbitos de las ciencias sociales, como la ciencia política y la sociología. Entre otras cosas, estos nuevos estudios han generado innovaciones importantes en el campo metodológico, como el desarrollo de colecciones importantes de historia oral y el trabajo con otro tipo de fuentes (fotografía, audiovisual, etc.). Además, han generado una reflexión importante sobre el papel de los Estados Nación en la producción de archivos. En algunos lugares, contribuyeron a estudiar el clima de polarización de los sesenta en la región. También aportaron a la caracterización de los regímenes autoritarios, al analizar sus dimensiones represivas vinculadas al terror y el miedo y las estrategias de consenso que sostuvieron 
dichos proyectos. Además, estas reflexiones pusieron una especial atención acerca de las tensiones que han estado en juego entre los discursos públicos sobre el pasado y la memoria histórica. Por último, en términos de intervención pública, estas investigaciones han contribuido a la reflexión sobre el respeto a los derechos humanos con relación a la política.

Aunque estos estudios trabajaron sobre procesos que fueron simultáneos en la región, mayoritariamente se desarrollaron concentrándose en la unidad nacional de análisis. Fue en mi experiencia como investigador del pasado reciente de Uruguay que me fui encontrando con estas redes de investigación, que evidenciaban esta sincronía de los procesos políticos. A comienzos de este siglo la experiencia del programa del Social Science Research Council, memorias de la represión, dirigido por la socióloga Elizabeth Jelin, fue particularmente rica en la posibilidad de desarrollar intercambios académicos sobre la región en torno al pasado reciente. Alrededor de 90 investigadores participamos, durante cuatro años, en un proyecto que se proponía estudiar las luchas por la memoria vinculadas al pasado de terrorismo de Estado y violencia política de la región. Dicho trabajo ha tenido impacto más allá de la región y ha servido como insumo para otros interesados en la relación entre memoria e historia con relación a episodios de violencia política y terrorismo estatal en lugares como México o España. Si bien esta red regional de investigadores y otras desarrollaron un rico intercambio sobre las similitudes de lo ocurrido, esto no se expresó en un trabajo que se enfocara en las conexiones de dichos procesos. A lo sumo, algunos desarrollaron trabajos de corte comparativo sobre el período. Pero fue en ese contexto que me empecé a interesar en las simultaneidades que evidenciaban diversos actores y coyunturas. Lo que parecía evidente era que, como consecuencia de la intensificación de la Guerra Fría latinoamericana, era posible encontrar una convergencia de los procesos políticos, que llevaba a que los mismos tuvieron una periodización similar, marcada por crisis democráticas en climas de polarización social y política, regímenes autoritarios con fuerte presencia militar, transiciones hacia democracias sin verdad y justicia y fuertes luchas centradas en la memoria, acerca de cómo incorporar esa experiencia en las presentes democracias. Además, dicha convergencia llevó a que una diversidad de actores tuvieran discursos y prácticas muy similares en la región.

Pero, en el caso de la Guerra Fría latinoamericana, hubo un elemento adicional, que reforzaba las tradicionales resistencias académicas nacionales a trabajar desde una perspectiva transnacional. Las argumentaciones políticas de la Guerra Fría explotaron al máximo la dicotomía (nacional-extranjero). Aunque este no sea el lugar para evaluar la influencia que cada uno de los actores internacionales tuvo en estos procesos nacionales, me interesa destacar las connotaciones negativas que se han desarrollado en torno a las conexiones entre actores domésticos y no-nacionales. Esto ciertamente ha impactado en las maneras en las que los asuntos vinculados a la violencia política han sido incorporados por las historiografías nacionales. Aunque los 
intercambios transnacionales fueron evidentes para cualquier analista de la región, y diversos actores (guerrilleros, militares, movimiento de derechos humanos, etc.) coordinaron regionalmente sus proyectos, estos procesos políticos han sido explicados más que nada en términos nacionales o por medio de enfoques comparativos que, en los últimos años, reforzaron las diferencias nacionales sin considerar los modos como fueron construidos los diálogos regionales.

Por otra parte, desde fines de siglo existió una creciente literatura sobre la Guerra Fría latinoamericana, producida en Estados Unidos. Dicha literatura se conjugaba con la renovación de los estudios sobre las dimensiones imperiales de la política exterior norteamericana, que desarrollada desde enfoques micro "zonas de contacto" y admitía una visión más compleja del fenómeno imperial (Joseph-Spenser, 2008).

Sin embargo, en la reflexión específica sobre el período que me interesa, la mirada que apuntaba a desarrollar una historia transnacional de la Guerra Fría latinoamericana parecía estar animada fundamentalmente por una reflexión sobre la política exterior norteamericana. Aunque las dimensiones locales han sido incluidas en varios trabajos, la reflexión apuntaba a evaluar las características de la política exterior norteamericana. Dos influyentes libros, como lo fueron The Last Colonial Massacre, de Greg Grandin, y Latin America's Cold War, de Hal Brands, aunque contradictorios entre sí, son ilustrativos del tipo de miradas que se desarrollaron durante estas últimas decadas para conceptualizar la Guerra Fría latinoamericana.

En el primer caso, Grandin, a través de una riquísima reconstrucción de diferentes historias de militantes políticos y sociales guatemaltecos, muestra cómo las dinámicas globales tuvieron un profundo impacto en la vida cotidiana de estos sectores populares. En sus capítulos existe un análisis muy fino de la intersección entre lo local y la política imperial. Pero en su conclusión, que fue lo más influyente de su libro, las dimensiones locales se pierden en una caracterización más general, que propone la dinámica revolución y contrarrevolución para entender el proceso político continental.

En el segundo caso, Brands realiza un desarrollo de diferentes procesos políticos latinoamericanos ilustrativos de la manera en que se vivió la Guerra Fría en el continente. En su trabajo, a través de la investigación de diferentes archivos localizados en varios países, repasa procesos políticos que han sido estudiados por historiadores nacionales y que simplemente son puestos en un contexto más amplio por Brands. Pero, a lo largo del libro, la pregunta central tiene que ver con la política exterior norteamericana, más allá de la presencia de actores locales.

Si bien estos enfoques se han acercado a pensar el lugar de América Latina en la Guerra Fría, su enfoque se ha orientado a pensar la política imperial y el lugar de la política local ha sido atendido de una manera subsidiaria o en relación a las resistencias o colaboración con Estados Unidos. Es en este marco que los enfoques establecen una relación dicotómica, 
donde de un lado se encuentra Estados Unidos y del otro, la región. No ha existido una mayor ponderación de las diferentes subregiones del continente, sus diferentes desarrollos políticos y económicos y las maneras particulares como se procesó la relación imperial a partir de dichas circunstancias. Por otro lado, esta perspectiva dicotómica opaca el papel de las relaciones y conflictos interregionales, que también fueron importantes en dicho proceso. En resumen, hay varios asuntos que fueron importantes en el proceso de la Guerra Fría latinoamericana que quedan al margen de esta literatura. Entre otras coas, porque, como plantea Andrew Kirkendall en un reciente artículo sobre la historiografía producida en Estados Unidos, la misma aún es depositaria de los propios intereses de Estados Unidos en aquel contexto, así como de los debates públicos en la sociedad norteamericana.

Mas allá de esta visión general, es cierto que algunos trabajos publicados en la primera década de este siglo resultaron buenos antecedentes para pensar lo local latinoamericano en el contexto global de la Guerra Fría, como el libro de Ariel Armony, Argentina, the United States, and the anti-communist crusade in Central America, 1977-1984, o el más reciente Beyond the Eagle's Shadow: New Histories of Latin America's Cold War, editado por Virginia Garrard-Burnett, Mark Atwood Lawrence y Julio E. Moreno. ${ }^{3}$

En un trabajo reciente, Tanya Harmer, además de continuar este tipo de aproximaciones estudiando, la política exterior de Salvador Allende en relación con Cuba, propone un enfoque metodológico innovador para pensar la Guerra Fría latinoamericana. Harmer plantea una perspectiva "multidimensional, comprehensive and descentralized" (Harmer, 2011), que involucra a varios actores: Brasil, Chile, Cuba, Estados Unidos y la Unión Soviética, así como a los partidos políticos de la izquierda chilena, la extrema derecha brasileña y una variedad de actores regionales. Todos estos grupos participaron en lo que ella llama el "sistema interamericano". Para Harmer, pensar en términos de sistema implica conceptualizar las relaciones multilaterales, más allá de las bilaterales. De esta manera, es claro que varios aspectos de las relaciones bilaterales entre Estados Unidos y México, Perú y, en particular, Brasil estuvieron fuertemente influidos por las políticas de Washington respecto del Chile de Salvador Allende. Además, en su enfoque sobre el sistema interamericano, no solamente importan los Estados, sino que existen actores no estatales, como grupos políticos vinculados a redes regionales, que también marcaron dichos procesos. En este sentido, la contribución de Harmer es importante porque ofrece una perspectiva para entender la particularidad de la dinámica política regional y para considerar la influencia de los actores no estatales en ese sistema.

Siguiendo a Harmer, creemos que esta perspectiva habilita a ampliar el pensamiento sobre el lugar de lo local en el orden global de la Guerra Fría. Pensar las relaciones dentro del sistema interamericano no implica abandonar o negar las condiciones estructurales imperiales 
sobre las cuales dicho sistema estaba construido. Pero sí implica entender, que entre el Imperio y el Estado Nación, hay múltiples espacios transnacionales que median, alteran y afectan los comportamientos de los actores locales, así como los del Imperio.

Dos ejemplos históricos clásicos pueden ilustrar mejor lo que estoy intentando decir. La creación de la Comisión Especial para América Latina (CEPAL), liderada por el economista argentino Raúl Prebisch en las Naciones Unidas, es un ejemplo de las ambiguas relaciones de ciertas elites latinoamericanas con los Estados Unidos. Estas relaciones ambiguas implicaron una tensión importante entre la teoría de la modernización, promovida por los Estados Unidos, y un incipiente pensamiento desarrollista, que se expandió en Latinoamérica en el contexto del crecimiento de las Industrias Sustitutivas de Importaciones. En los setenta, las dictaduras del Cono Sur comenzaron a desarrollar prácticas represivas coordinadas, en lo que se dio en Ilamar la Operación Cóndor, que las llevaron a acciones represivas regionales, como el asesinato o la captura de personas fuera de todo marco legal en los países vecinos. Luego, el plan se extendió a Estados Unidos, con el asesinato de Orlando Letelier en Washington, y a otras acciones conducidas en Europa. Dicha actuación coordinada, marcada por una perspectiva muy fundamentalista acerca de los significados de la lucha contra la subversión, llevó a un conflicto importante con los Estados Unidos durante la presidencia de Jimmy Carter.

Los dos ejemplos dan cuenta de que las relaciones entre Estados Unidos y las elites nacionales fueron importantes, pero también de que, además, existieron otros espacios transnacionales que mediaron entre dichas relaciones. Dichos espacios fueron fundamentales para entender fenómenos tan importantes en la Guerra Fría latinoamericana como el desarrollismo y la doctrina de la seguridad nacional. Este espacio transnacional tiene que ver con el sistema interamericano, pero por momentos lo trasciende, como lo muestran los defensores de la CEPAL en la ONU o los militares de Cóndor desarrollando acciones en Europa. Por ello, es ahí donde podemos encontrar varias claves para entender la Guerra Fría latinoamericana.

Creo que es en ese espacio transnacional donde un diálogo entre la historia reciente producida en los escenarios nacionales y los cold war studies podría ser positivo. El diálogo entre ambas tradiciones académicas, que hablan de un mismo periodo histórico, pero que lo miran desde diferentes lugares, podría otorgarles una mayor densidad global a ambos enfoques. A continuación, propondré algunos temas que, desde mi punto de vista, tienen cierta potencialidad para dicho diálogo en la historia de la segunda mitad del siglo XX. La selección será relativamente arbitraria y tiene que ver con las temáticas que mayormente he frecuentado. Espero que estos casos ejemplifiquen el espíritu de encuentro que estoy sugiriendo.

El desarrollismo latinoamericano ha sido una palabra nativa del continente. Para los investigadores sociales de la región, el concepto remite a una tradición latinoamericana que fue 
fuertemente influyente en los cincuentas y sesentas. Dicha influencia no fue solo relevante en el campo de las políticas económicas, sino que tuvo dimensiones que las trascendieron. Existió un desarrollismo político, que ambientó movimientos políticos de masas. Existió una cultura desarrollista, que alentó industrias culturales y proyectos urbanísticos tan vanguardistas como Brasilia. Por último, el desarrollismo tuvo un fuerte impacto en las ciencias sociales latinoamericanas y en las maneras como dichas ciencias sociales influenciaron en los procesos políticos de la región. Sin embargo, existe una creciente literatura que indaga sobre el impacto de la teoría de la modernización y el desarrollo en los países del Tercer Mundo mirando a estos fenómenos como externos a la región. Trabajos como el de Michael Latham exploraron en las maneras que, desde Estados Unidos, intelectuales, técnicos e instituciones internacionales promovieron determinadas nociones de modernización y desarrollo durante la segunda mitad del siglo XX (Latham, 2000).

Estos enfoques parecen descuidar que existió una historia propia de dichas nociones, que tuvieron diferentes relaciones con las ideas que venían del norte. Una serie de investigaciones localizadas y que miran el impacto del desarrollismo e incluso la teoría de la modernización desde el punto de vista local muestran cómo dicho pensamiento habilitó muy diversas trayectorias, algunas cercanas al objetivo de promover una modernidad neocolonial, pero otras fuertemente críticas del papel de los centros de poder internacionales, así como de los sectores dominantes a nivel nacional. El trabajo de Ricardo López muestra la trayectoria de radicalización de sectores técnicos de la clase media colombiana, que en la década de los cincuentas se comprometieron con la modernización del país y comenzaron a constatar que los principales opositores a la misma eran los sectores oligárquicos. Esta investigación ejemplifica los ambiguos impactos que estas ideologías de lo moderno tuvieron en diversos sectores medios y populares en el continente (López, 2014). Similares trayectorias son rastreables en diversos intelectuales latinoamericanos, tales como Paulo Freire o Raúl Prebisch. Así lo muestran sus recientes biografías históricas (Kirkendall, 2008) (Dosman, 2008). Lo mismo puede decirse de la comunidad de cientistas sociales mayoritariamente formados en la sociología de la modernización, que terminan en el dependentismo a fines de los sesentas (Blanco, 2010). Mientras desde el centro la teoría de la modernización y el desarrollismo fueron vistos meramente como la incorporación de una práctica neoimperial, desde lo local y lo regional podemos percibir que efectivamente funcionó como una reformulación neocolonial para algunos, pero para otros fue la plataforma desde la cual se comenzaron argumentos que explican la radicalización de los sectores medios y de la academia a mediados de los sesentas.

A nivel local, en cada uno de los países del Cono Sur ha habido un creciente interés histórico y periodístico por las izquierdas que emergieron en las décadas de los sesentas y setentas. Dicho interés no solo remite al pasado de aquellas décadas, sino que, de alguna 
manera, aquellas experiencias ayudan a una mejor comprensión de fenómenos más duraderos, que explican cómo las izquierdas latinoamericanas sobrevivieron a la Posguerra Fría y lograron una visibilidad a nivel global durante la primera década del siglo XXI, cuando las ideas de izquierda estaban en franca caída en otras zonas del mundo.

La producción es diversa en su calidad, pero en varios casos ha implicado una renovación en los enfoques (historia cultural, intelectual, política), así como en el trabajo heurístico que realizan estos investigadores. Un importante número de esfuerzos vinculado a las colecciones de historia oral, así como a la formación de archivos, estuvo asociado a la preocupación por la reconstrucción histórica de la izquierda. En varios países del Cono Sur, el estudio de estos grupos es tan abundante que ha habilitado al desarrollo de jornadas, revistas especializadas y archivos. Más allá de la diversidad de enfoques, en la gran mayoría de ellos primó una perspectiva fundamentalmente política, que priorizó los estudios de las ideas, estrategias y prácticas de estas organizaciones, a diferencia del tipo de enfoques culturales o vinculados a preocupaciones subalternistas, que tienen mayor influencia en el norte (Carnovale, 2011), (Leibner, 2011), (Ridenti y Aarão Reis, 2007), (Rey Tristán, 2006).

Más allá del valor de dicha producción historiográfica, existió un elemento paradójico en su desarrollo. Varios de esos movimientos políticos e intelectuales estuvieron asociados a tradiciones internacionalistas construidas en la primera mitad del siglo, y otros a un creciente latinoamericanismo asociado a estrategias culturales y políticas continentales impulsadas desde Cuba. El crecimiento de ambas corrientes también se desarrolló en un evidente diálogo con los sesentas globales. Sin embargo, más allá de estas dimensiones internacionalistas y transnacionales, los estudiosos de las izquierdas latinoamericanas mayoritariamente han quedado atrapados por el Estado Nación como unidad de análisis. Pensar a las izquierdas en el marco de un diálogo regional e internacional ayudaría a una mejor comprensión de la constitución y de las diversas estrategias tomadas en cada momento. Ayudaría a mostrar cómo no todos los comportamientos son el resultado de las ideologías, sino que la interpretación de la experiencia regional tuvo consecuencias en los comportamientos políticos. La región fue el laboratorio desde el cual se extrajeron las categorías necesarias para construir un marco interpretativo común acerca de la realidad política local. De cada evento local los militantes sacaron conclusiones generales, que tuvieron consecuencias en las luchas por venir.

Además, el diálogo entre estos estudios locales de la izquierda con la historia global podría ayudar a repensar la geografía de los global sixties. Así como ocurrió con las revoluciones del siglo XIX, el 68 fue conceptualizado mayoritariamente a partir de la centralidad de Europa occidental y Estados Unidos. La gran mayoría de los trabajos reconocen el rol del Tercer Mundo y sus luchas en la agitación desarrollada en el Primer Mundo, pero estos aspectos 
quedan limitados a una mera influencia y no constituyen parte de una misma red de circulación de ideas y actores. Sin embargo, los hechos que tuvieron lugar en Europa y Estados Unidos también recibieron la influencia de lo que ocurrió en Latinoamérica.

Uno de los iconos más populares en los países centrales durante el 68 fue la imagen del Che Guevara. Más allá de las reminiscencias románticas de la trayectoria de Guevara, dicha imagen ilustraba el peso de la experiencia histórica reciente de América Latina en las ideas y estrategias políticas que alentaron la revuelta global. Los sesentas fueron más globales que su narrativa histórica, pero gran parte de la reflexión parece opacar el activo papel de las periferias en las ideas y repertorios de protesta de los países centrales. En ese sentido, podemos decir que el estudio de estas experiencias locales puede ayudar a comprender mejor la globalidad de esa década, reintegrar el papel de los procesos que han sido subestimados por la bibliografía de la historia global.

La historia de la reflexión sobre los regímenes dictatoriales es más matizada en relación a las tensiones entre los enfoques locales y aquellos que los trascienden. A fines de los setentas los primeros debates académicos sobre las dictaduras fueron desarrollados desde una perspectiva regional que reconocía las características comunes de dicho ascenso autoritario en la región. El exilio académico y el vínculo con instituciones universitarias del norte (Estados Unidos, México, Europa) fueron desarrollando una serie de redes transnacionales, a través de las cuales se fueron construyendo las primeras categorías para pensar estos regímenes. Aunque categorías como Estado Burocrático Autoritario y Terrorismo de Estado surgen inicialmente para el estudio del caso argentino, rápidamente comenzaron a ser utilizadas para pensar otras dictaduras de la región. Por otra parte, en las últimas décadas, una serie de estudios desarrollados desde el periodismo y las ciencias sociales han indagado en las dimensiones transnacionales de las políticas represivas. Existe una abundante bibliografía sobre el ya mencionado Plan Cóndor, que muestra la articulación regional de las dictaduras, el impacto que la doctrina de la seguridad nacional tuvo en la reformulación de los ejércitos nacionales y la respuesta articulada regionalmente, presentándose como una vanguardia en la "Tercera Guerra Mundial" contra el comunismo frente a las críticas del Gobierno de Jimmy Carter.

Mas allá de estos enfoques, en las últimas dos décadas ha existido un desarrollo importante de historiadores que, desde las historias nacionales, han propuesto maneras innovadoras de abordar el fenómeno del autoritarismo en la región. Estas investigaciones se pueden agrupar en dos campos. Por un lado, aquellas que han trabajado en torno a las dimensiones civiles del autoritarismo. Los trabajos de Marina Franco (2012), en Argentina, o de Álvaro Rico (2005), en Uruguay, han señalado las continuidades de ciertas prácticas e ideas autoritarias entre los regímenes democráticos y las dictaduras. Mientras tanto, otros investigadores como Magdalena 
Broquetas y Verónica Valdivia, han explorado en la constitución de nuevos actores de derecha, que rompen con las tradiciones previas, y que se anticipan a las demandas autoritarias (Broquetas, 2014). Además, otros autores han explorado en nuevas miradas para entender el fenómeno autoritario y trascender las dimensiones represivas de estos regímenes. A través del estudio de políticas específicas, como las sociales, educativas, culturales y de comunicación, así como del diálogo del régimen con actores de la sociedad civil, dichos historiadores buscan entender las estrategias de las dictaduras, en algunos casos efectivas, para obtener cierto nivel de consenso en las sociedades conosureñas durante el autoritarismo (Rollemberg-Viz Quadrat, 2010).

Aunque dichos historiadores han participado de redes regionales de debate sobre dichos asuntos, estos intercambios aún no han tenido resultados en nuevas líneas de investigación que enfoquen estas temáticas desde una perspectiva transnacional. Creemos que poner a estos actores civiles en un diálogo regional, así como en otros espacios de la Guerra Fría global, ayudaría a entender mejor su proceso de renovación en el contexto de la Guerra Fría, así como las dimensiones autoritarias que el liberalismo democrático de la Guerra Fría contuvo. Gran parte de las argumentaciones teórico-políticas vinculadas al Estado de excepción, que son tan recurrentes en la contemporánea "Guerra Contra el Terrorismo", tienen antecedentes evidentes en los autoritarismos conosureños de los setentas. Poner estos regímenes en un contexto global ayudaría a pensar de una manera más crítica acerca de los límites de la experiencia de la democrática occidental de la segunda mitad del siglo XX durante la Guerra Fría.

La reflexión sobre el movimiento de derechos humanos ha sido la más desarrollada en su dimensión transnacional. Como Samuel Moyn (2010) muestra en su libro, el resurgimiento de la noción de derechos humanos en el contexto global de la década de los setentas tuvo mucho que ver con los movimientos de solidaridad con las violaciones a los derechos humanos cometidas por las dictaduras, junto a las denuncias sobre lo que ocurría en Europa oriental. Es interesante que, si comparamos los enfoques que se han desarrollado sobre la región, vemos cierta tensión en el cómo se conceptualiza el diálogo transnacional. Mientras que el trabajo de Vania Markarian (2005), Left in Transformation: Uruguayan Exiles and the Latin American Human Rights Networks, pone un énfasis mayor en la apropiación local y la reinterpretación de los derechos humanos por parte los militantes de izquierda uruguayos, el trabajo de James Green (2010), We Cannot Remain Silent: Opposition to the Brazilian Military Dictatorship in the United States, resalta el papel de los activistas norteamericanos en dicho desarrollo. Las perspectivas de ambos trabajos dan cuenta de los posibles enfoques que puedan tener una lectura transnacional del surgimiento de dichos movimientos. En ellas, las tensiones norte sur siguen presentes. El reciente trabajo The Justice Cascade: How Human Rights Prosecutions Are Changing World Politics, de Kathryn Sikkink (2011), da cuenta del lugar que el Cono Sur, particularmente Argentina, tuvo en 
el movimiento de derechos humanos global. Estos trabajos dejan muchas preguntas abiertas acerca de cuál fue la relación entre estos movimientos locales y las redes transnacionales. Asimismo, abren una reflexión que trasciende el pasado reciente y que se pregunta cuál es la relación entre las versiones locales de las nociones de derechos humanos vinculadas a la declaración de 1948 y los procesos globales de re conceptualización en los setentas.

Por último, otra línea de investigación histórica que dialoga con los derechos humanos se refiere a los estudios sobre memoria colectiva desarrollados durante la primera década del siglo XXI por el proyecto Memorias de la represión, dirigido por Elizabeth Jelin (2002). Dicho programa implicó una innovación historiográfica en la manera de estudiar las tensiones entre memoria e historia, que lo diferenció de los modelos sugeridos por la escuela francesa. Aunque dichos trabajos se desarrollaron en un diálogo académico regional, la gran mayoría de las investigaciones se dieron sobre unidades locales y nacionales. Lo transnacional parece una tarea pendiente, que es sugerida por gran parte de los trabajos.

\section{A MODO DE CIERRE}

$\mathrm{L}$ o que he intentado en este repaso es proponer un diálogo entre diferentes campos académicos: el de los estudios de la Guerra Fría realizados en los Estados Unidos y aquellos trabajos producidos por las historiografías nacionales, en lo que se ha dado en llamar la historia reciente. Partí de la idea de que los estudios producidos en el norte prestaban una mayor atención al lugar de la potencia imperial, mientras que los historiadores recientes trabajaron sobre las dinámicas ocurridas dentro de la unidad de análisis del Estado Nación. En varios casos, las trayectorias de los propios investigadores trabajando en ambos lugares, 0 preocupándose por fenómenos locales desde el norte, muestran que la división no resulta tan clara. Sin embargo, dicha división resulta útil para mostrar que existen diferentes perspectivas, o miradas desde las cuales se puede hacer historia global. Importa poco si el que escribe está en el norte 0 en el sur. Pero la perspectiva desde la cual se está haciendo la pregunta y con qué tradiciones historiográficas dialoga es relevante. En este sentido, creo que una mayor apertura de las historiografías nacionales podría enriquecerlas, así como interpelar ciertas miradas de los fenómenos globales, cuyas narrativas navegan por los canales de las estructuras que construyeron los países centrales. En última instancia, se trata de invertir el sentido común académico, que asume que lo que se produce en los estados nacionales del sur es local y lo que se produce en el norte es global, para reflexionar sobre las condiciones locales que influyen en la perspectiva global del norte y para asumir la perspectiva global como necesidad para un mejor entendimiento de las historias nacionales en el sur. 


\section{Notas}

1 A modo de ejemplo, Schensmmaier plantea que paradójicamente a contrapelo del interés por lo global en Estados Unidos la proporción de trabajos traducidos ha descendido de 8,6 a 3 \% (Schensmmaier, 2011: 40).

2 A modo de ejemplo, ver McMahom (1994 y 2003).

3 Ver también: Calandra; Franco (2012) y Harmer; Riquelme (2014).

\section{BiBLIOGRAFIA}

ARMONY, Ariel. Argentina, the United States, and the anti-communist crusade in Central America, 1977-1984. Athens: Ohio University Center for International Studies, 1997.

BLANCO, Alejandro.Ciencias sociales en el cono sur y la génesis de una nueva elite intelectual (1940-1965). In: ALTAMIRANO, Carlos (director) Historia de los intelectuales en América Latina. Tomo II. Buenos Aires: Katz Editores, 2010.

BRANDS, Hal. Latin America's Cold War. Cambridge: Harvard University Press, 2010.

BROQUETAS, Magdalena. La trama autoritaria: derechas y violencia en Uruguay (1958-1966). Montevideo: Banda Oriental, 2014.

CALANDRA, Benedetta; FRANCO, Marina. La guerra fría cultural en América Latina: desafíos y límites para una nueva mirada de las relaciones interamericanas. Buenos Aires : Biblos, 2012.

CARNOVALE, Vera. Los combatientes: historia del PRT-ERP. Buenos Aires: Siglo Veintiuno Editores, 2011.

CONRAD, Sebastian. What is global history? Princeton: Princeton, 2015.

DOSMAN, Edgar J. The life and times of Raúl Prebisch, 1901-1986. Mc Gill Queens University Press, 2008.

FRANCO, Marina. El exilio: argentinos en Francia durante la dictadura. Buenos Aires: Siglo Veintiuno Editores, 2008.

. Un enemigo para la nación: orden interno, violencia y "subversión", 1973-1976. Buenos Aires: Fondo de Cultura Económica, 2012.

GARRARD-BURNETT, V., ATWOOD LAWRENCE, M., y MORENO, J. Beyond the eagle's shadow: new histories of Latin America's Cold War. USA: New Mexico Press, 2013.

GRANDIN, Greg. The last colonial massacre: Latin America in the Cold War. Chicago: University of Chicago Press, 2004.

GREEN. James. We cannot remain silent: opposition to the Brazilian Military Dictatorship in the United States. Durham, NC: Duke University Press, 2010.

HARMER, Tanya. Allende's Chile and the Inter-American Cold War. Chapel Hill: University of North Carolina Press, 2011.

_- RIQUELME, Alfredo. Chile y la Guerra Fría global, Santiago de Chile: RIL Editores, 2014.

JELIN, Elizabeth. Los trabajos de la memoria. Madrid : Siglo XXI de España Editores/ Social Science Research Council, 2002. 
JOSEPH, G. M.; SPENSER Daniela. In from the cold: Latin America's new encounter with the Cold War. Durham: Duke University Press, 2008.

KIRKENDALL, Andrew J. Cold War Latin America: The State of the Field. H-Diplo Essay no. 119.

—. Paulo Freire \& the cold war politics of literacy. Chapel Hill: The University of North Carolina Press, 2010.

LATHAM, Michael E. Modernization as ideology: American social science and "nation building" in the Kennedy era. Chapel Hill: University of North Carolina Press, 2000.

LEIBNER, Gerardo. Camaradas y compañeros. Una historia politica y social de los comunistas del Uruguay. Montevideo: Trilce, 2011.

LOPEZ, Ricardo. Una democracia musculosa. Identificaciones profesionales, lucha de clases y la radicalización política de la clase media en Bogotá, 1958-1965. Contemporánea. Historia y problemas del siglo XX, año 5, volumen 5, 2014.

MARKARIAN, Vania. Left in transformation: Uruguayan exiles and the Latin American human rights networks, 1967-1984. New York: Routledge, 2005.

MC MAHON, Robert J. The Cold War on the periphery. The United States, India, and Pakistan. Columbia University Press, 1994.

—. Cold War. A very short introduction. Cornwall: Oxford, 2003.

MIGNOLO, Walter D. La idea de América Latina. La herida colonial y la opción decolonial. Barcelona: Gedisa, 2007. MOYN, Samuel. The last utopia, Human rights in History. Cambridge: Harvard University Press, 2010.

REY TRISTÁN, Eduardo. A la vuelta de la esquina: la izquierda revolucionaria uruguaya, 1955-1973. Montevideo: Editorial Fin de Siglo, 2006.

RICO, Alvaro. Cómo nos domina la clase gobernante: orden político y obediencia social en la democracia posdictadura Uruguay (1985-2005). Montevideo: Ediciones Trilce, 2005.

RIDENTI, Marcelo y AARAO REIS, Daniel (comp.). História do marxismo no Brasil. Campinas: Unicamp, 2007.

ROLLEMBERG, Denise. Exillio: entre raizes e radares. Rio de Janeiro: Editora Record, 1999.

; VIZ QUADRAT, Samantha. A construção social dos regimes autoritários, Legitimidade, consenso e consentimento no século XX. Brasil e América Latina. Rio de Janeiro: Civilização Brasileira, 2010.

SCHENSMMAIER, Dominic. Global perspectives on Global History. Theories and approaches in a connected world. New York: Cambridge University Press, 2011.

SIKKINK, Kathryin. The Justice cascade: how human rights prosecutions are changing world politic. New York: W.W. Norton \& Co., 2011.

VALDIVIA, Verónica. Nacionales y gremialistas: el "parto" de la nueva derecha política chilena, 1964-1973. Santiago: Lom Ediciones, 2008.

WESTAD, Odd Arn. The global Cold War: third world interventions and the making of our times. Cambridge/ New York: Cambridge University Press, 2005. 